\title{
Journal of Bone and Mineral Metabolism Best Paper Award 2015
}

Published online: 16 October 2015

(C) The Japanese Society for Bone and Mineral Research and Springer Japan 2015

The Journal of Bone and Mineral Metabolism Best Paper Award was established in 2008. Candidates for the award must be members of the Japanese Society of Bone and Mineral Research, and the winner is honored at the Society's Annual Meeting.

We are pleased to announce that the following article has received JBMM Best Paper Award.

"FGF and ERK signaling coordinately regulate mineralization-related genes and play essential roles in osteocyte differentiation" by

Ai Kyono, Nanthawan Avishai, Zhufeng Ouyang,

Gary E. Landreth, Shunichi Murakami

J Bone Miner Metab (2012) 30:19-30

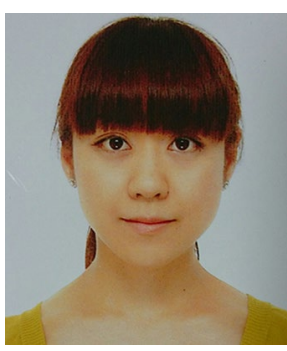

Dr. Ai Kyono

\begin{abstract}
To examine the roles of FGF and ERK MAPK signaling in osteocyte differentiation and function, we performed microarray analyses using the osteocyte cell line MLO-Y4. This experiment identified a number of mineralization-related genes that were regulated by FGF2 in an ERK MAPK-dependent manner. Real-time PCR analysis indicated that FGF2 upregulates Ank, Enppl, Mgp, Slc20al, and Dmpl in MLO-Y4 cells. Consistent with this observation, the selective FGF receptor inhibitor PD173074 decreased Ank, Enppl, Slc20a1, and Dmpl mRNA expression in mouse calvaria in organ culture. Since Dmp1 plays a central role in osteocyte differentiation and mineral homeostasis, we further analyzed FGF regulation of Dmp1. Similar to FGF2, FGF23 upregulated Dmp1 expression in MLO-Y4 cells in the presence of Klotho. Furthermore, increased extracellular phosphate levels partially inhibited FGF2-induced upregulation of Dmpl mRNA expression, suggesting a coordinated regulation of Dmpl expression by FGF signaling and extracellular phosphate. In MLO-Y4 osteocytes and in MC3T3E1 and primary calvaria osteoblasts, U0126 strongly inhibited both basal expression of Dmpl mRNA and FGF2-induced upregulation. Consistent with the in vitro observations, real-time PCR and immunohistochemical analysis showed a strong decrease in Dmp1 expression in the skeletal elements of $E R K 1^{-1-}$; ERK2 $2^{f l o x f f l o x}$; Prx1-Cre mice. Furthermore, scanning electron microscopic analysis revealed that no osteocytes with characteristic dendritic processes develop in the limbs of $E R K 1^{-1-} ; E R K 2^{f l o x}$ ffox $;$ Prx 1-Cre mice. Collectively, our observations indicate that FGF signaling coordinately regulates mineralization-related genes in the osteoblast lineage and that ERK signaling is essential for Dmpl expression and osteocyte differentiation.
\end{abstract}

We offer our sincere congratulations on behalf of the Journal of Bone and Mineral Metabolism, with best wishes for further development of the author's research.

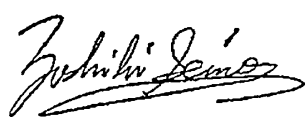

Yoshiki Seino

Editor-in-Chief

Journal of Bone and Mineral Metabolism 